\title{
Connection between the Principles of Thermodynamics and the Conservation Laws: Physical Meaning of the Principles of Thermodynamics
}

\author{
L. I. Petrova \\ MSU Faculty of Computational Mathematics and Cybernetics, Moskva, Russia \\ Email: ptr@cs.msu.su
}

How to cite this paper: Petrova, L.I. (2018) Connection between the Principles of Thermodynamics and the Conservation Laws: Physical Meaning of the Principles of Thermodynamics. Journal of Applied Mathematics and Physics, 6, 2697-2704. https://doi.org/10.4236/jamp.2018.612223

Received: November 28, 2018

Accepted: December 26, 2018

Published: December 29, 2018

Copyright $\odot 2018$ by author(s) and Scientific Research Publishing Inc. This work is licensed under the Creative Commons Attribution International License (CC BY 4.0).

http://creativecommons.org/licenses/by/4.0/

\begin{abstract}
It has been shown that the first principle of thermodynamics follows from the conservation laws for energy and linear momentum. And the second principle of thermodynamics follows from the first principle of thermodynamics under realization of the integrating factor (namely, temperature) and is a conservation law. The significance of the first principle of thermodynamics consists in the fact that it specifies the thermodynamic system state, which depends on interaction between conservation laws and is non-equilibrium due to a non-commutativity of conservation laws. The realization of the second principle of thermodynamics points to a transition of the thermodynamic system state into a locally-equilibrium state. Phase transitions are examples of such transitions.
\end{abstract}

\section{Keywords}

Skew-Symmetric Differential Forms, Conservation Laws, First Principle of Thermodynamics, Realization of Integrating Factor, The Second Principle of Thermodynamics, The Entropy

\section{Introduction}

During the process of science evolution the concept of "conservation laws" in thermodynamics, physics and mechanics was taken different senses. In mathematical physics, which describes material systems such as the thermodynamic and gas-dynamic systems, the systems of charged particles, and others, the concept of "conservation laws" relates to the conservation laws for energy, linear momentum, angular momentum, and mass that establish the balance between 
variations of physical quantities and external action. These are conservation laws that are described by differential equations or by the equations in terms of increments of physical quantities and governing variables. From here on they will be referred as the balance conservation laws.

In the field theory and in the theoretical mechanics "the conservation laws" are those that claim an existence of conservative physical quantities or objects. Such conservation laws are described by closed exterior skew-symmetric forms. The Noether theorem can serve as an example of such conservation laws formulation, which, under some conditions, can be written as $d \omega=0$. Below such conservation laws will be referred to as "exact" ones.

In thermodynamics the conservation laws are associated with the principles of thermodynamics.

That is; the concept of "conservation laws" is connected with the balance conservation laws, exact conservation laws and some regularities; the example of which are the principles of thermodynamics.

It turns out that there exists a connection between these representations of the conservation laws [1] [2]. The exact conservation laws are obtained from the balance conservation laws as a result of interactions of the balance conservation laws between them. The first principle of thermodynamics is just an example of such interaction (mutual influence).

Below it will be shown that the first principle of thermodynamics relates to the balance conservation law for energy and linear momentum that appears to be non-commutative and describe the non-equilibrium state of thermodynamic system. The second principle of thermodynamics follows discretely from the first one as the result of interaction of balance conservation laws under a realization of the integrating factor (the inverse temperature). That corresponds to an exact conservation law (the existence of entropy as a state function) and describes the transition of thermodynamic system to the locally-equilibrium state that is accompanied by emergence of some fluctuations.

\section{Connection the Thermodynamic Principles with Conservation Laws}

\subsection{Connection between the First Principle of Thermodynamics and Conservation Laws}

The first principle of thermodynamics can be written as [3]: $d E+\delta w=\delta Q$, here $d E$ is the change of the thermodynamic system energy, $\delta w$ is the work done by the system (this means that $\delta w$ is expressed in terms of the system parameters), $\delta Q$ is the amount of the heat influx into the system (i.e. the external action onto the system). Since the term $\delta w$ can be expressed in terms of the system parameters and specifies a real (rather than virtual) change, it can be designated by $d w$, and hence, the first principle of thermodynamics takes the form

$$
d E+d w=\delta Q
$$


Here it should be emphasized a certain indeterminacy: the left-hand side of relation (1) consists of differentials, whereas the right-hand side includes an expression that is not a differential. Below it will be shown that such an indeterminacy is not an inaccuracy and this has a physical meaning.

What is a difference between the first principle of thermodynamics and the balance conservation laws?

For thermodynamic system the balance conservation law of energy can be written as

$$
d E=\delta Q+\delta G
$$

where by $\delta G$ we designate the energetic actions with the exception of heat influx. For thermodynamic system the balance conservation law for linear momentum (the change of linear momentum of the system in its dependence on the force mechanical action onto the system) can be written as

$$
d w=\delta W
$$

Here $\delta W$ stands for the force (mechanic) action onto the system (an external compression of the system, an influence of boundaries and so on).

If to combine relations (2) and (3), one can obtain the relation

$$
d E+d w=\delta Q+\delta G+\delta W
$$

that is just the evolutionary relation for the thermodynamic system.

By comparing relation (4) followed from the balance conservation laws for energy and linear momentum, and relation (1), one can see that they coincide if the heat influx is the only external action onto the thermodynamic system ( $\delta W=0$ and $\delta G=0)$.

Thus, the first principle of thermodynamics follows from the balance conservation laws for energy and linear momentum.

Below it will be shown that the second principle of thermodynamics relates to the first principle of thermodynamics. However, this connection is not identical. The second principle of thermodynamics is obeyed discretely. This principle follows from the first principle of thermodynamics under realization (during the process of varying the thermodynamic system state) of the integrating factor (namely, the inverse temperature).

This follows from relation (1) that describes the first principle of thermodynamics.

The right-hand side of relation (1) contains the expressions $\delta Q$ that describe a heat influx into the system, i.e. the external action onto the system. This expression is not a potential one and, hence, cannot be a differential. Thus it appears that relation (1), which corresponds to the first principle of thermodynamics, includes a term that that is not a differential. Such a relation cannot be integrated identically. This points out to the fact that the quantity describing a heat influx cannot directly convert into thermodynamic quantities. The actions, which are not potential ones, cannot directly be converted into the parameters of the thermodynamics system itself, into the energy or the momentum of the 
thermodynamic system elements, for example.

Since the heat influx cannot directly convert into the parameters of the thermodynamics system itself, it is accumulated as a certain quantity that in termodynamic system acts as a certain force that converts the thermodynamics system into a non-equilibrium state.

Thus it appears that the first principle of thermodynamics specifies the thermodynamic system state. From this principle it follows that the thermodynamic system state is non-equilibrium. To this fact it points out an absence of the state function.

\subsection{Connection the Second Principle of Thermodynamics with Conservation Laws}

The relation (1), which corresponds to the first principle of thermodynamics, describes also the transitions of thermodynamic system from non-equilibrium state to the locally-equilibrium state. This discloses a physical meaning of the second principle of thermodynamics.

Relation (1) links differentials and the expression that is not a differential, that is, the relation (1) is not an identical one.

From the properties of skew-symmetric differential forms it follows that under degenerate transformations from nonidentical relations it can be obtained the identical relation, namely, a relation that consists of differentials only. From such a relation one can obtain the state function, and this will point to a locally-equilibrium state of thermodynamic system.

The expression in the right-hand side of relation (1) is a skew-symmetric form. But this is an unclosed form, i.e. a skew-symmetric form with nonzero differential (for this reason such skew-symmetric form is not a differential).

For the identical relation be realized from nonidentical relation, it is necessary that from unclosed skew-symmetric form (which differential is nonzero) in the right-hand side of nonidentical relation the closed skew-symmetric form (which differential equals zero and hence it is a differential), be realized. This is possible only under degenerate transformation, namely, a transformation that does not conserve a differential. Such degenerate transformation is allowed only at additional condition that can be realized if there exist any degrees of freedom.

In the case of thermodynamic system the realization of integrating factor may serve as a realization of the degenerate transformation condition.

If the condition of degenerate transformation be satisfied, from the nonidentical relation, which corresponds to the first principle of thermodynamics, it follows an identical relation that corresponds to the second principle of thermodynamics.

Let us consider the case when the work performed by the system is carried out through the compression. Then $d w=p d V$ (here $p$ is the pressure and $V$ is the volume) and $d E+d w=d E+p d V$. As it is known, the form $d E+p d V$ can become a differential if there is the integrating factor $\theta$ (a quantity which depends only on the system parameters), where $1 / \theta=p V / R$ is called the 
temperature $T[3]$.

In this case the form $(d E+p d V) / T$ turns out to be a differential (interior) of some quantity that referred to as entropy $S$ :

$$
(d E+p d V) / T=d S
$$

although the form $d E+d w$ consists of differentials, in the general case without the integrating factor it is not a differential due to the fact that its terms depend on different variables, namely, the first term is determined by variables that specifies the internal structure of elements, and the second term depends on variables that specify an interaction between elements, for example, the pressure.

If the integrating factor $\theta=1 / T$ has been realized, that is, relation (5) proves to be satisfied, from relation (1), which corresponds to the first principle of thermodynamics, it follows

$$
\delta Q / T=d S
$$

This is just the second principle of thermodynamics for reversible processes.

From relation (5) one can see that, under realization of integrating factor, the differentials of energy and linear momentum made up a differential, that is, the thermodynamic potential. From this it follows that entropy $S$ becomes a state function. And its value can be found from the second principle of thermodynamics (using relation (6)).

Relation (6), which corresponds to the second principle of thermodynamics, takes place when the heat influx is the only action onto the system. In the case when the system experiences a certain mechanical action, in the right-hand side of relation (6) it may appear the term related to the mechanical action. In this case the condition of degenerate transformation, in particular, the integrating factor, may not be realized. In this case the entropy proves to be a functional rather than a state function. You can see the work [4] on entropy.

The realization of the entropy differential means that the exact conservation law is obeyed. A differential of the entropy differential equals zero, that is, the entropy differential is a conservative quantity. It turns out that the second principle of thermodynamics is an exact conservation law in the case when the equality is fulfilled.

Thus, the first principle of thermodynamics follows from the balance conservation laws for energy and linear momentum, and the second principle of thermodynamics follows from the first one under the fulfillment of the integrability condition i.e. a realization of the integrating factor (the inverse temperature) and is an exact conservation law.

Here it should be emphasized that the second principle of thermodynamics, unlike the first principle of thermodynamics, fulfills only discretely, namely, only under realization of integrating factor.

In the above analyzed case the differential of entropy (rather than entropy itself) becomes a closed form (that is, a conservative quantity). In this case entropy manifests itself as the thermodynamic potential, namely, the function of 
state.

For entropy be a closed form itself, i.e. a conservative quantity (the differential of closed form is equal to zero), one more condition has to be realized. Such a condition could be the realization of the integrating direction, an example of that is the speed of sound: $a^{2}=\partial p / \partial \rho=\gamma p / \rho$. In this case it is valid the equality $d s=d\left(p / \rho^{\lambda}\right)=0$ from which it follows that entropy $s=p / \rho^{\lambda}=$ const is a closed form (of zero degree). However it does not mean that a state of the thermodynamic system is identically isoentropic. Entropy is a constant only along the integrating direction (for example, on the adiabatic curve or on the front of the sound wave), whereas in the direction normal to the integrating direction the normal derivative of entropy has a break.

Under realization of the integrating direction the transition from the variables $E$ and $V$ to the variables $p$ and $\rho$ is a degenerate transformation.

It is worthy to underline that both temperature and the speed of sound are not continuous thermodynamic variables. They are variables that are realized in the thermodynamic processes if the thermodynamic system has any degrees of freedom. One can see the analogy between the inverse temperature and the speed of sound: the inverse temperature is the integrating factor and the speed of sound is the integrating direction.

\section{Physical Meaning of the Principles of Thermodynamics}

In subsection 2 it was pointed out that the first principle of thermodynamics specifies the material system state. From the first principle of thermodynamics it follows that the thermodynamic system state is non-equilibrium one. The absence of the state function points to the non-equilibrium state of the thermodynamic system.

From the second principle of thermodynamics it follows that under realization of temperature, which depends only on parameters of thermodynamic system (and the realization of relevant integrating factor), the transition to the equilibrium system state is possible. To this fact it points the realization of entropy as a state function. But, the equilibrium state can be realized only locally as it follows from the second principle of thermodynamics, which, as it was already noted, is fulfilled only discretely. The phase transitions are examples of this phenomenon. In this case the total state of thermodynamic system remains to be non-equilibrium, and this fact follows from the first principle of thermodynamics.

The first principle of thermodynamics, as it has been shown, describes the in teraction between conservation laws for energy and momentum that appear to be noncommutative. Due to the non-commutativity of the conservation laws, the actions, to which the thermodynamics system undergoes, cannot directly convert into the quantities of the thermodynamics system itself. They convert into a certain unmeasurable quantity that acts like an internal force which induces the non-equilibrium system state. The example of such unmeasurable quantity is a quantity that is described by the commutator of unclosed form 
$d E+d w$.

As the second principle of thermodynamics points out, the transition to a locally-equilibrium state (under realization of integrating factor or other integrating conditions caused by any degrees of freedom) relates to the transition of unmeasurable quantity acted as an internal force into the characteristics of the material system itself. This reveals in the form of emerging any formations such as fluctuations.

Note that in actual processes a total state of the thermodynamic system is non-equilibrium one and the commutator of the form $d E+d w$ is nonzero. A quantity that is described by a commutator and acts as an internal force can grow if the integrating conditions are not realized.

It is a cause of development of instability in the thermodynamic system. Such an explanation for the development of thermodynamic instability was proposed by Prigogine in his paper [5] (he was awarded the Nobel prize for this work). As it has been shown by Prigogine, the development of instability in thermodynamic systems and the entropy increase in irreversible processes are due to production of so called "excess entropy". The commutator just describes such "excess entropy". However, Prigogine did not explained the mechanism of emergence of thermodynamic fluctuations and other thermodynamic formations.

A closed static system, if left to its own devices, can tend to a state of total thermodynamic equilibrium. This corresponds to the system functional tendency to its asymptotic maximum. In the dynamical system the tendency of the system to a state of total thermodynamic equilibrium can be violated by dynamical processes and transitions to a state of local equilibrium.

\section{Conclusions}

Thus, one can see that the first principle of thermodynamics follows from the balance conservation laws for energy and linear momentum. And the second principle of thermodynamics follows discretely from the first one under a realization of the integrating factor (the inverse temperature) and is an exact conservation law.

The principles of thermodynamics describe the thermodynamic system state and the processes of transition of thermodynamic system from non-equilibrium state into the locally-equilibrium one (the phase transitions are examples) that is accompanied by the emergence of fluctuations and other observable formations.

\section{Conflicts of Interest}

The author declares no conflicts of interest regarding the publication of this paper.

\section{References}

[1] Petrova, L. (2013) Role of the Conservation Laws in Evolutionary Processes and 
Generation of Physical Structures. American Journal of Modern Physics, 2, 104-110.

[2] Petrova, L.I. (2012) The Noncommutativity of the Conservation Laws: Mechanism of Origination of Vorticity and Turbulence. International Journal of Theoretical and Mathematical Physics, 2, 84-90.

[3] Haywood, R.W. (1980) Equilibrium Thermodynamics. John Wiley \& Sons, Hoboken.

[4] Petrova, L.I. (2012) Physical Meaning and a Duality of Concepts of Wave Function, Action Functional, Entropy, the Pointing Vector, the Einstein Tensor. Journal of Mathematics Research, 4, 78-88.

[5] Prigogine, I. (1955) Introduction to Thermodynamics of Irreversible Processes. Charles C Thomas Publisher, Springfield. 